\title{
Virtual Compton Scattering at Low Energy and the Generalized Polarizabilities of the nucleon
}

\author{
H.Fonvieille \\ Laboratoire de Physique Corpusculaire IN2P3-CNRS \\ University Blaise Pascal Clermont-II, 63177 AUBIERE, France
}

April 27, 2018

\begin{abstract}
Virtual Compton Scattering on the nucleon: $\gamma^{*} N \rightarrow \gamma N$ is a new and rapidly developing field at low and high energies, with the emergence of recent concepts such as Generalized Parton Distributions (GPDs) at high energy, and Generalized Polarizabilities (GPs) at low energy. This lecture is about the low energy part, i.e. for energies in the $(\gamma p)$ center-of-mass mainly up to the $\Delta(1232)$ resonance region. I review the concept of GPs and the experiments dedicated to their measurement.
\end{abstract}

\section{The Generalized Polarizabilities of the Nucleon}

The formalism of Polarizabilities in Real Compton Scattering (RCS) and Generalized Polarizabilities in Virtual Compton Scattering (VCS) has been the subject of previous lectures at the Erice School of Nuclear Physics by Nicole D'Hose. I refer the reader to her very detailed and pedagogical lecture on the subject [1]. I will summarize the concepts again, and will concentrate on some recent experimental developments in the field.

\subsection{The physical meaning of Generalized Polarizabilities}

The GPs are new observables to study nucleon structure. They have a natural connection with two well-known processes: RCS and electron-nucleon elastic scattering.

1. From $Q^{2}=0$ to finite $Q^{2}$ : when going from RCS : $\gamma N \rightarrow \gamma N$ to VCS : $\gamma^{*} N \rightarrow \gamma N$ at photon virtuality $Q^{2}$, the electromagnetic polarizabilities of the nucleon become functions of $Q^{2}$ and are called the Generalized Polarizabilities. This concept was first introduced in 1995 [2] and allows to probe the polarizability locally inside the nucleon, with a distance scale given by $Q^{2}$. For example, in most models the electric GP $\alpha_{E}$ is predicted to decrease monotonically with $Q^{2}$, while the magnetic GP $\beta_{M}$ is predicted to go through a maximum before decreasing, as shown in figure 1. This last feature is usually explained by the dominance of diamagnetism due to the pion cloud at long distance, or small $Q^{2}$, and the dominance of paramagnetism due to a quark core at short distance, or large $Q^{2}$. In VCS there are also new GPs due to the longitudinal polarization state of the virtual photon. 

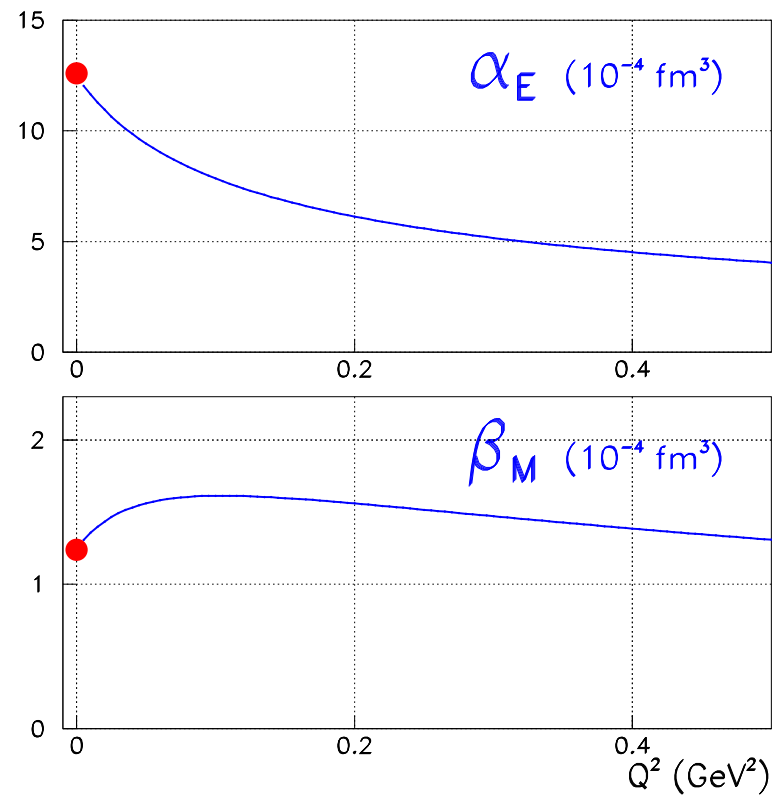

Figure 1: The electric and magnetic GPs of the proton as calculated in heavy baryon chiral perturbation theory [6]. The magnitude $\left(10^{-4} \mathrm{fm}^{3}\right)$ reminds us that the nucleon is a strongly bound and very rigid object.

2. From finite $q^{\prime}$ to $q^{\prime}=0$ : similarly to the polarizabilities in RCS, the GPs in VCS are theoretically defined in the limit q' $\rightarrow 0$, where q' is the energy of the final real photon. This is the "zero-frequency" limit corresponding to a static electromagnetic field. In this limit, from kinematic point of view the VCS process $\gamma^{*} N \rightarrow \gamma N$ becomes the process $\gamma^{*} N \rightarrow N$, i.e. elastic electron-nucleon scattering. As stated in ref. [3], "VCS at threshold can be interpreted as electron scattering by a target which is in constant electric and magnetic fields". The GPs can then be seen as Fourier transform of densities of electric charges and magnetization of a nucleon deformed by an applied EM field.

\subsection{The VCS amplitude and its multipole decomposition}

VCS is accessed experimentally by exclusive photon electroproduction as shown in Figure 2. The main kinematic variables are the CM three-momenta $q_{c m}$ and $q_{c m}^{\prime}$ of the initial and final photons, and the CM angles of the outgoing real photon w.r.t. $\vec{q}_{c m}$ : the polar angle $\theta_{C M}$ and azimuthal angle $\varphi$ (see fig. 2). Other useful variables are the virtual photon four-momentum transfer squared $Q^{2}$ and its polarization rate $\epsilon$.

Due to electron scattering, one also has the Bethe-Heitler process $(\mathrm{BH})$ where the final photon is emitted by the incoming or outgoing electron. Figure 3] shows the decomposition of the photon electroproduction amplitude $T$ into the coherent sum of the BH, Born and Non-Born parts:

$$
T_{e p \rightarrow e p \gamma}=T_{B H}+T_{V C S \text { Born }}+T_{V C S \text { Non-Born }}
$$

The $(\mathrm{BH})$ and (VCS Born) parts are known and entirely calculable, with the nucleon EM form factors as inputs. The Non-Born amplitude contains the unknown part that one aims to measure. The 

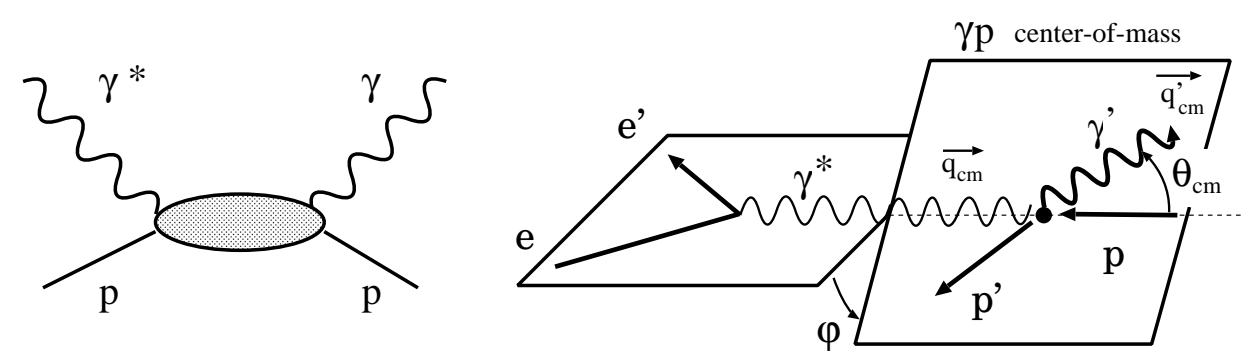

Figure 2: The VCS graph on the proton and the main kinematic variables of the process.

interest is that this part describes the excited spectrum of the nucleon in the intermediate state, the excitation and de-excitation being specifically of electromagnetic origin. ${ }^{1}$

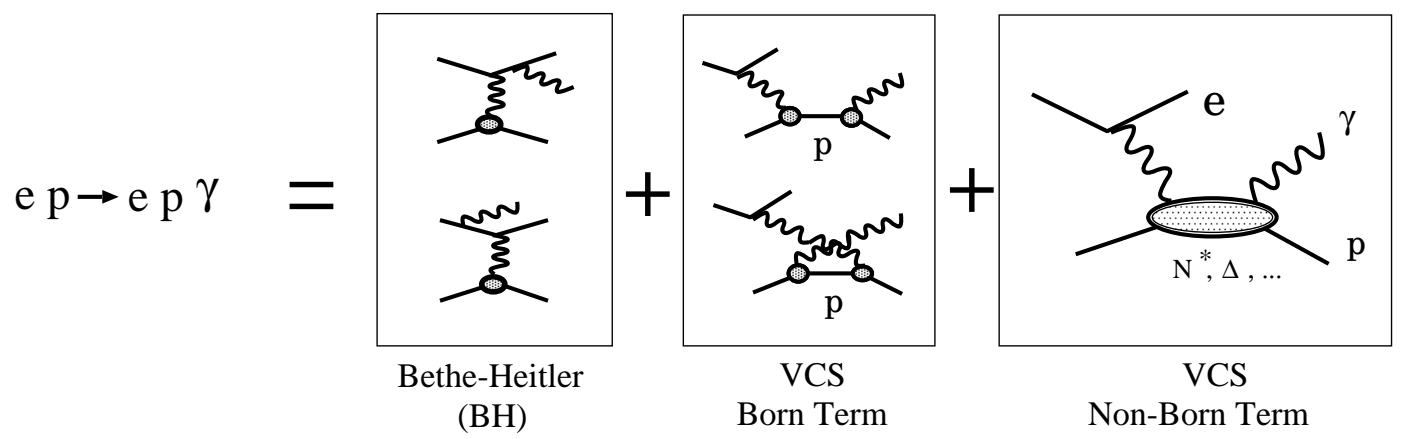

Figure 3: Decomposition of the photon electroproduction amplitude.

The GPs are introduced using the decomposition of the VCS Non-Born amplitude [2] into multipoles corresponding to well-defined initial and final EM transitions. A multipole $H$ is characterized by five quantum numbers: $L, L^{\prime}=$ (orbital) angular momentum of the initial and final photons; $\rho, \rho^{\prime}=$ their polarisation states $(\rho=0,1,2$ for longitudinal, magnetic, electric); $S=1$ (resp.0) allows for nucleon spin-flip (resp. non spin-flip) in the process. A polarizability $P$ is defined in the zero-frequency limit by:

$$
\mathrm{GP}=\left.P^{\left(\rho^{\prime} L^{\prime}, \rho L\right) S}\left(q_{c m}\right) \quad \sim \quad \lim \right|_{q_{c m}^{\prime} \rightarrow 0}\left(\frac{1}{q_{c m}^{L^{\prime}}} \frac{1}{q_{c m}^{L}} H_{N o n B o r n}^{\left(\rho^{\prime} L^{\prime}, \rho L\right) S}\left(q_{c m}, q_{c m}^{\prime}\right)\right)
$$

The GPs are a function of $q_{c m}$, or equivalently $Q^{2}{ }^{2}$ The lowest order is $L^{\prime}=1$, leading finally to six independent (dipole) GPs after application of nucleon crossing symmetry and charge conjugation invariance [4, 5]. The choice of the six GPs is not unique; a standard set is given in table 11. The two scalar GPs $(S=0)$ are a generalization of the electric and magnetic polarizabilities $\alpha_{E}$ and $\beta_{M}$. Among the four spin GPs $(S=1)$ some have a correspondence in RCS.

Most models of nucleon structure at low energy give predictions for these new observables: heavy baryon chiral perturbation theory [6, 7], linear sigma model [8], effective lagrangian model [9], dispersion

\footnotetext{
${ }^{1}$ It should be noted that the definition of the Born and Non-Born terms of the VCS amplitude is not unique [5]. In ref. 2] the Non-Born part includes the $\pi^{0} t$-channel exchange graph, and the Born terms include the antinucleon graph.

${ }^{2}$ This four-momentum transfer is actually the photon virtuality when $q_{c m}^{\prime} \rightarrow 0$, at fixed $q_{c m}$ and $\epsilon$. It is given by : $\tilde{Q}^{2}=2 M_{N} \cdot\left(\sqrt{M_{N}^{2}+q_{c m}^{2}}-M_{N}\right)$. One also defines the virtual photon $\mathrm{CM}$ energy in the same limit : $\tilde{q}_{0}=$ $M_{N}-\sqrt{M_{N}^{2}+q_{c m}^{2}}[3]$.
} 
Table 1: The six lowest-order GPs. Notations use either the EM transitions or the five indices. $S=0$ (resp. 1) correspond to scalar (resp. spin) GPs.

\begin{tabular}{|c|c|c|c|c|c|c|}
\hline final $\gamma$ & initial $\gamma^{*}$ & $\mathrm{~S}$ & $P^{\left(\rho^{\prime} L^{\prime}, \rho L\right) S}\left(q_{c m}\right)$ & $P^{X \rightarrow Y}$ & $\begin{array}{c}\text { RCS limit } \\
\left(Q^{2}=0\right)\end{array}$ & $\begin{array}{c}\text { resonances } \\
\text { involved }\end{array}$ \\
\hline E1 & $\mathrm{C} 1$ & 0 & $P^{(01,01) 0}$ & $P^{C 1 \rightarrow E 1}$ & $-\frac{4 \pi}{e^{2}} \sqrt{\frac{2}{3}} \alpha_{E}$ & $D_{13}, S_{11}$ \\
M1 & M1 & 0 & $P^{(11,11) 0}$ & $P^{M 1 \rightarrow M 1}$ & $-\frac{4 \pi}{e^{2}} \sqrt{\frac{8}{3}} \beta_{M}$ & $P_{33}, P_{11}$ \\
E1 & $\mathrm{C} 1$ & 1 & $P^{(01,01) 1}$ & $P^{C 1 \rightarrow E 1}$ & 0 & $D_{13}, S_{11}$ \\
M1 & M1 & 1 & $P^{(11,11) 1}$ & $P^{M 1 \rightarrow M 1}$ & 0 & $P_{33}, P_{11}$ \\
E1 & M2 & 1 & $P^{(01,12) 1}$ & $P^{M 2 \rightarrow E 1}$ & $-\frac{4 \pi}{e^{2}} \frac{\sqrt{2}}{3} \gamma_{3}$ & $D_{13}$ \\
M1 & $\mathrm{C} 2$ & 1 & $P^{(11,02) 1}$ & $P^{C 2 \rightarrow M 1}$ & $-\frac{4 \pi}{e^{2}} \sqrt{\frac{8}{27}}\left(\gamma_{2}+\gamma_{4}\right)$ & $P_{33}$ \\
\hline
\end{tabular}

relation model [10, 11, non-relativistic constituent quark models [12, 13, etc. A review of the theoretical predictions can be found in ref. [13. Ideally, one aims at measuring the whole set of the six GPs as a function of $Q^{2}$ to make the most complete comparison with theory.

\subsection{The pion threshold and methods to extract the GPs}

The pion threshold is defined by $W=m_{N}+m_{\pi}$ where $W$ is the total energy in the $(\gamma p)$ center-ofmass. Above this threshold the VCS amplitude becomes complex. While $T_{B H}$ and $T_{V C S}$ Born remain real, the amplitude $T_{V C S}$ Non-Born acquires an imaginary part, due to the coupling to the $\pi N$ channel. Figure 4 shows an example of $(e p \rightarrow e p \gamma)$ cross section as a function of $W$. Below pion threshold, the effect of GPs, which is contained in $d \sigma_{N o n-B o r n}$, is small (10-15\% maximum). It becomes important in the region of the $\Delta(1232)$ resonance. It is worth noting that the GPs are defined at $q_{c m}^{\prime}=0$ but their contribution to the cross section is zero at this point (see eq. 33). Therefore measurements must be done at finite $q_{c m}^{\prime}$.

There are presently two methods to extract GPs from measurements of (unpolarized) photon electroproduction cross sections. The first method uses a Low Energy Theorem (LET) and is valid below pion threshold only. The second method is based on the Dispersion Relation (DR) approach and its domain of validity includes the $\Delta(1232)$ resonance, up to the $N \pi \pi$ threshold.

\subsection{The Low Energy Theorem}

The Low Energy Theorem [14] was first applied to VCS by P.Guichon et al. [2]. It leads to the following expression for the unpolarized $e p \rightarrow e p \gamma$ cross section below pion threshold :

$$
\begin{aligned}
d^{5} \sigma_{E X P} & =d^{5} \sigma_{B H+B o r n}+\left[q_{c m}^{\prime} \phi \Psi_{0}+\mathcal{O}\left(q_{c m}^{\prime 2}\right)\right] \\
\Psi_{0} & =v_{1} \cdot\left(P_{L L}-\frac{1}{\epsilon} P_{T T}\right)+v_{2} \cdot P_{L T}
\end{aligned}
$$

where $\phi, v_{1}, v_{2}$ are kinematic coefficients defined in [3]. The bracket on the right-hand side is a lowenergy expansion (LEX) in $q_{c m}^{\prime}$. The unknown part of the nucleon structure is contained in the $\Psi_{0}$ term (dipole GPs) and higher order terms (higher-order polarizabilities). The three structure functions $P_{L L}, P_{T T}$ and $P_{L T}$ are the following combinations of the lowest-order GPs: 


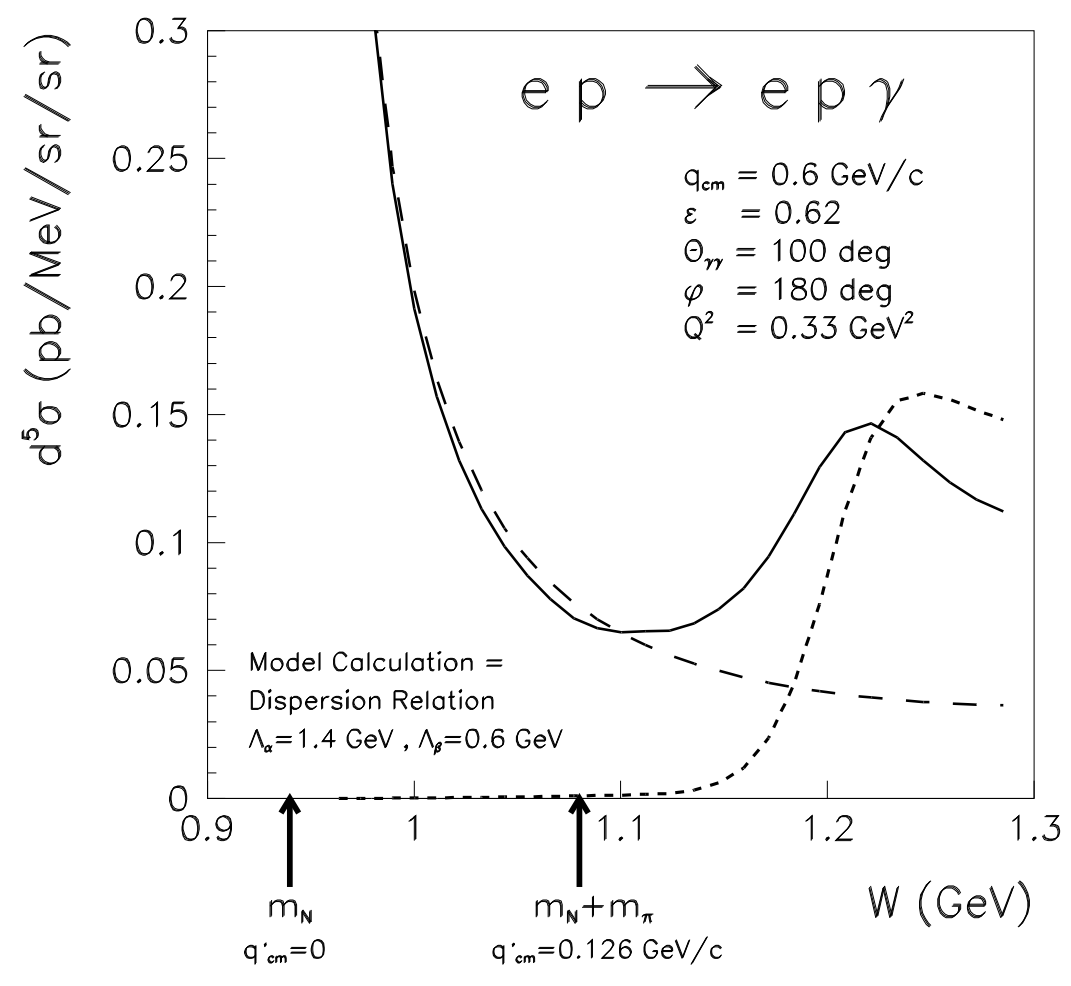

Figure 4: Example of photon electroproduction cross section $d^{5} \sigma / d k_{l a b}^{\prime} d \Omega_{e}^{\prime} d \Omega_{p C M} \quad\left(k_{l a b}^{\prime}=\right.$ scattered electron energy, $d \Omega_{e}^{\prime}=$ scattered electron lab solid angle, and $d \Omega_{p C M}=$ final proton $\mathrm{CM}$ solid angle). Contributions of BH+Born (long-dashed), Non-Born (short-dashed) and total cross section (solid).

$$
\begin{aligned}
& P_{L L}\left(q_{c m}\right)=-2 \sqrt{6} M_{N} G_{E} P^{(01,01) 0}\left(q_{c m}\right) \\
& P_{T T}\left(q_{c m}\right)=-3 G_{M} \frac{q_{c m}^{2}}{\tilde{q}_{0}} \times\left[P^{(11,11) 1}\left(q_{c m}\right)-\sqrt{2} \tilde{q}_{0} P^{(01,12) 1}\left(q_{c m}\right)\right] \\
& P_{L T}\left(q_{c m}\right)=\sqrt{\frac{3}{2}} \frac{M_{N} q_{c m}}{\tilde{Q}} G_{E} P^{(11,11) 0}\left(q_{c m}\right)+\frac{3}{2} \frac{\tilde{Q} q_{c m}}{\tilde{q}_{0}} G_{M} P^{(01,01) 1}\left(q_{c m}\right)
\end{aligned}
$$

The electric and magnetic form factors $G_{E}, G_{M}$ are taken at four-momentum transfer squared $\tilde{Q}^{2}$ (see footnote 2). These equations tell us that $P_{L L}$ is proportional to the electric GP $\alpha_{E}, P_{T T}$ is a combination of two spin GPs, and $P_{L T}$ is a combination of the magnetic GP $\beta_{M}$ and a spin GP.

In an experimental analysis based on the LET, one compares the measured cross section $d^{5} \sigma_{E X P}$ to the $d^{5} \sigma_{B H+B o r n}$ cross section, entirely calculable from QED. At $q_{c m}^{\prime}=0$ these two cross sections coincide, as shown by eq. $3 .{ }^{3}$ The $\Psi_{0}$ term is obtained by extrapolation of the quantity $\Delta \mathcal{M}=$ $\left(d^{5} \sigma_{E X P}-d^{5} \sigma_{B H+B o r n}\right) /\left(q_{c m}^{\prime} \phi\right)$ to $q_{c m}^{\prime}=0$. In the experiments performed so far, the data for $\Delta \mathcal{M}$ do not exhibit any significant dependence in $q_{c m}^{\prime}$, so the extrapolation to $q_{c m}^{\prime}=0$ is done by a simple average. This is done in bins in the outgoing photon angles $\theta_{C M}$ and $\varphi$, at fixed $\epsilon$ and $q_{\mathrm{cm}}$. The

\footnotetext{
${ }^{3}$ In the term $q_{c m}^{\prime} \phi$ multiplying $\Psi_{0}$ in eq. 3 the factor $\phi$ remains finite when $q_{c m}^{\prime} \rightarrow 0$.
} 
resulting $\Psi_{0}$ term is then fitted as a linear combination of two free parameters, which are the structure functions $P_{L L}-P_{T T} / \epsilon$ and $P_{L T}$.

Figure 5 shows an example of the angular dependence of photon electroproduction cross section, for in-plane and out-of-plane kinematics. The polarizabilities are responsible for the difference between the full and dashed curves. In-plane, the pattern of the cross section and of the polarizability effect is rather complicated, due to the strong BH-VCS interference. The most suitable region to measure GPs is away from the BH peaks (located by the $e$ and $e^{\prime}$ arrows on the graph). Out-of-plane, the cross section behaves much more smoothly and the GP effect is almost constant.
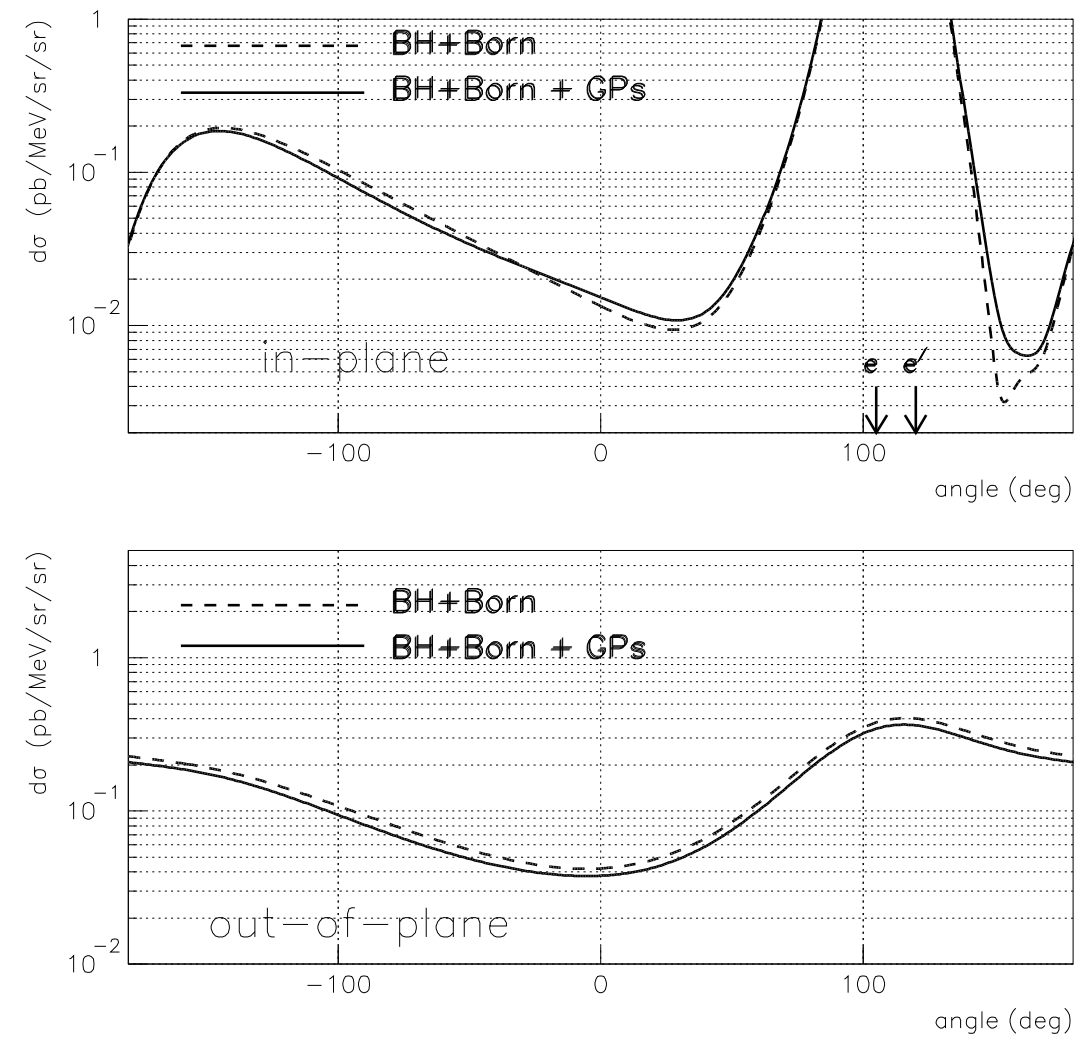

Figure 5: $(e p \rightarrow e p \gamma)$ cross section at $q_{c m}=1080 \mathrm{MeV} / \mathrm{c}, q_{c m}^{\prime}=105 \mathrm{MeV} / \mathrm{c}, \epsilon=0.95, \varphi=$ 0 or $180^{\circ}$ (top) or out-of-plane (bottom). In abscissa is the longitude angle of the outgoing photon when the polar axis is oriented perpendicular to $\vec{q}_{c m}$. Top plot: longitude=0 when $\theta_{C M}=0$. Bottom plot: latitude $=45^{\circ}$. Dashed curve $=\mathrm{BH}+$ Born, solid curve $=\mathrm{BH}+\mathrm{Born}$ + a realistic first order GP effect.

\subsection{The Dispersion Relation Model for VCS}

B.Pasquini et al. developed a model for Real and Virtual Compton Scattering based on Dispersion Relations 10, 11. This approach has several advantages, including a rather large range of validity in $Q^{2}$, and an applicability in the $\Delta(1232)$ resonance region, where the LET does not hold. In this model the VCS Non-Born amplitudes are given by dispersive integrals. They can be split in two parts: 1) a " $\pi N$ " part which describes the $\pi N$ intermediate states, and is calculated using MAID 
pion photoproduction amplitudes [15]; 2) a part due to asymptotic behavior plus contributions beyond " $\pi N$ ". The spin GPs are entirely predicted, but not the two scalar GPs. The asymptotic behavior of $\alpha_{E}\left(Q^{2}\right)$ and $\beta_{M}\left(Q^{2}\right)$ has to be parametrized, and for that purpose a dipole form has been chosen:

$$
\alpha_{E}\left(Q^{2}\right)-\alpha_{E}^{\pi N}\left(Q^{2}\right)=\frac{\left[\alpha_{E}^{e x p}-\alpha_{E}^{\pi N}\right] Q^{2}=0}{\left(1+Q^{2} / \Lambda_{\alpha}^{2}\right)^{2}}
$$

(same relation for $\beta$ with parameter $\left.\Lambda_{\beta}\right) . \alpha_{E}^{\pi N}\left(\beta_{M}^{\pi N}\right)$ is the $\pi N$ dispersive contribution evaluated from MAID [15], $\alpha_{E}^{\exp }\left(\beta_{M}^{\exp }\right)$ is the experimental value of the polarizability at $Q^{2}=0$ [16], and the mass coefficients $\Lambda_{\alpha}$ and $\Lambda_{\beta}$ are the two free parameters to be fitted from experiment.

In an experimental analysis based on the DR model, one compares the measured cross section $d^{5} \sigma_{E X P}$ to the one predicted by the model for several values of $\Lambda_{\alpha}$ and $\Lambda_{\beta}$. The fitted values of the parameters are the ones which minimize the $\chi^{2}$ between experimental and theoretical cross sections. 4 Then it is straightforward to determine the scalar GPs by use of eq. 5. and also if desired the structure functions $P_{L L}, P_{T T}$ and $P_{L T}$.

\section{The unpolarized experiments}

Table 2 summarizes the three first VCS experiments which have been dedicated to the determination of GPs, at MAMI, the Thomas Jefferson National Accelerator Facility (JLab) and MIT-Bates. They have measured unpolarized $(e p \rightarrow e p \gamma)$ cross sections and extracted GPs and structure functions by the methods cited above.

Table 2: The first dedicated VCS experiments.

\begin{tabular}{|c|c|c|c|c|c|}
\hline Lab & $Q^{2}\left(\mathrm{GeV}^{2}\right)$ & CM energy W & $\epsilon$ & data taking & status \\
\hline MAMI-A1 VCS & 0.33 & $<\left(m_{N}+m_{\pi}\right)$ & 0.62 & $1995+97$ & publ.2000 [17] \\
JLab E93-050 & $1.0,1.9$ & up to $1.9 \mathrm{GeV}$ & $0.95,0.88$ & 1998 & publ.2004 [18, 19] \\
Bates E97-03 & 0.05 & $<\left(m_{N}+m_{\pi}\right)$ & 0.90 & 2000 & in analysis [20] \\
\hline
\end{tabular}

These experiments detect the outgoing electron and proton in magnetic spectrometers. High resolution is crucial, since the outgoing photon is reconstructed as the missing particle. High luminosities are also required, due to the smallness of photon electroproduction cross sections. A high duty cycle is necessary to minimize the rate of accidental coincidences. Below pion threshold, the elastic process $(e p \rightarrow e p)$ is kinematically close to VCS and may induce background in the detectors. Finally, absolute cross sections must be determined accurately in order to extract the small effect due to the polarizabilities. The acceptance of the apparatus must be calculated with great care by a Monte-Carlo simulation [21]. For all these reasons, such experiments are difficult.

The main sources of systematic errors arise from: energy and angle calibration of the spectrometers, luminosity calculation, uncertainty in the radiative corrections to photon electroproduction [22], the choice of form factors in the (BH+Born) cross section, and also possible cross section shape departures from fit hypothesis.

\footnotetext{
${ }^{4}$ Such fits are performed locally in $Q^{2}$, so the result is practically insensitive to the somewhat arbitrary dipole form chosen for $\alpha_{E}\left(Q^{2}\right)-\alpha_{E}^{\pi N}\left(Q^{2}\right)$ and $\beta_{M}\left(Q^{2}\right)-\beta_{M}^{\pi N}\left(Q^{2}\right)$.
} 


\subsection{The experimental results from LEX analyses}

Figure [6 is a visual representation of the fit performed on the $\Psi_{0}$ term of eq. 3, in the MAMI [17] and JLab [18] experiments. The polarizability signal shows up clearly, in the non-zero slope $\left(P_{L L}-P_{T T} / \epsilon\right)$ and intercept $\left(P_{L T}\right)$ of the fitted straight line.

MAMI $/ Q 2=0.33 \mathrm{GeV} 2$

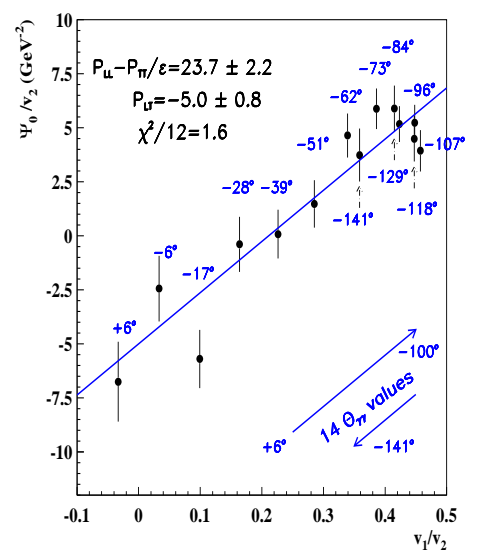

$\mathrm{JLAB} / \mathrm{Q} 2=0.92 \mathrm{GeV} 2$

$\mathrm{JLab} / \mathrm{Q} 2=1.76 \mathrm{GeV} 2$

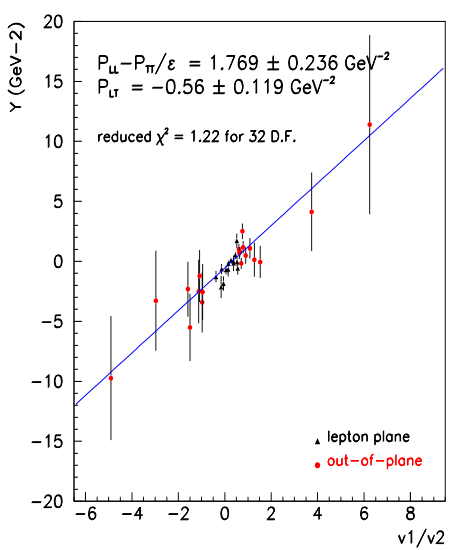

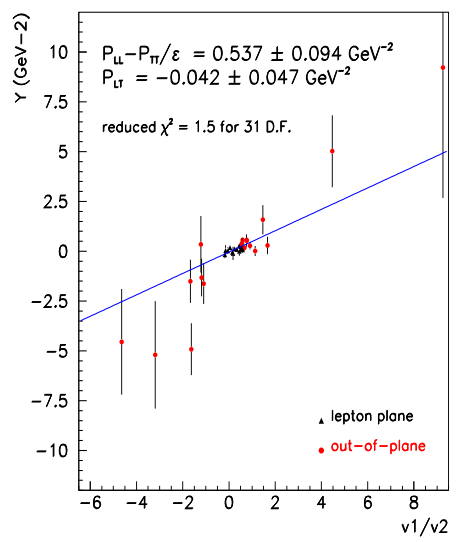

Figure 6: The polarizability fit of the LEX analyses of the MAMI and JLab experiments. In ordinate: $\Psi_{0} / v_{2}=P_{L T}+\frac{v_{1}}{v_{2}}\left(P_{L L}-P_{T T} / \epsilon\right)$ (cf.eq. 3).

The LEX result of MAMI is at low $Q^{2}$ and therefore has been compared with most theoretical model predictions of the VCS structure functions [1]. As a summary, the values of $\left(P_{L L}-P_{T T} / \epsilon\right)$ and $\left(P_{L T}\right)$ measured by this experiment agree well with the calculation of heavy baryon chiral perturbation theory (HBChPT) 23, 24], while they disagree with all other model predictions. The HBChPT calculation is done at order $p^{3}$. The calculation at next order $p^{4}$ is not fully available yet, it is published only for the spin GPs [7].

At higher $Q^{2}$, the LEX result of JLab cannot be compared to these low energy models. The first statement one can deduce from the numerical values reported in figure 6 is that the structure functions definitely show a strong fall-off with four-momentum transfer. A more complete discussion is postponed to the next section, in connection with the DR model.

\subsection{The experimental results from DR analyses}

The Jlab VCS data has been analyzed using the DR approach as explained in section 1.5. The mass coefficients $\Lambda_{\alpha}$ and $\Lambda_{\beta}$ which parametrize the behavior of the asymptotic contribution to the GPs $\alpha_{E}$ and $\beta_{M}$ have been fitted on experimental photon electroproduction cross sections. This has been done for three different data sets, covering different values of $Q^{2}\left(0.9\right.$ and $\left.1.8 \mathrm{GeV}^{2}\right)$ and $\mathrm{W}$ (below pion threshold and up to $1.3 \mathrm{GeV}$ ). The range of values found is $[0.70-0.77] \mathrm{GeV}$ for $\Lambda_{\alpha}$ and $[0.63-0.79] \mathrm{GeV}$ for $\Lambda_{\beta}$ [18]. This indicates that the asymptotic contribution to the scalar GPs falls off more rapidly than the standard dipole elastic form factor (having $\Lambda=0.84 \mathrm{GeV}$ ).

In such an analysis the determination of the electric and magnetic GPs is straightforward, from eq. 5. Figure 7 shows the world results for these GPs deduced from the MAMI and JLab experiments, including LEX and DR analyses. To make this figure, the LEX results, initially in terms of structure functions, have been translated in terms of the electric and magnetic GPs by use of eq. (4. In these 
equations we took the spin GPs as predicted by the DR model, and the proton EM form factors were calculated using the parametrization of ref. [25].

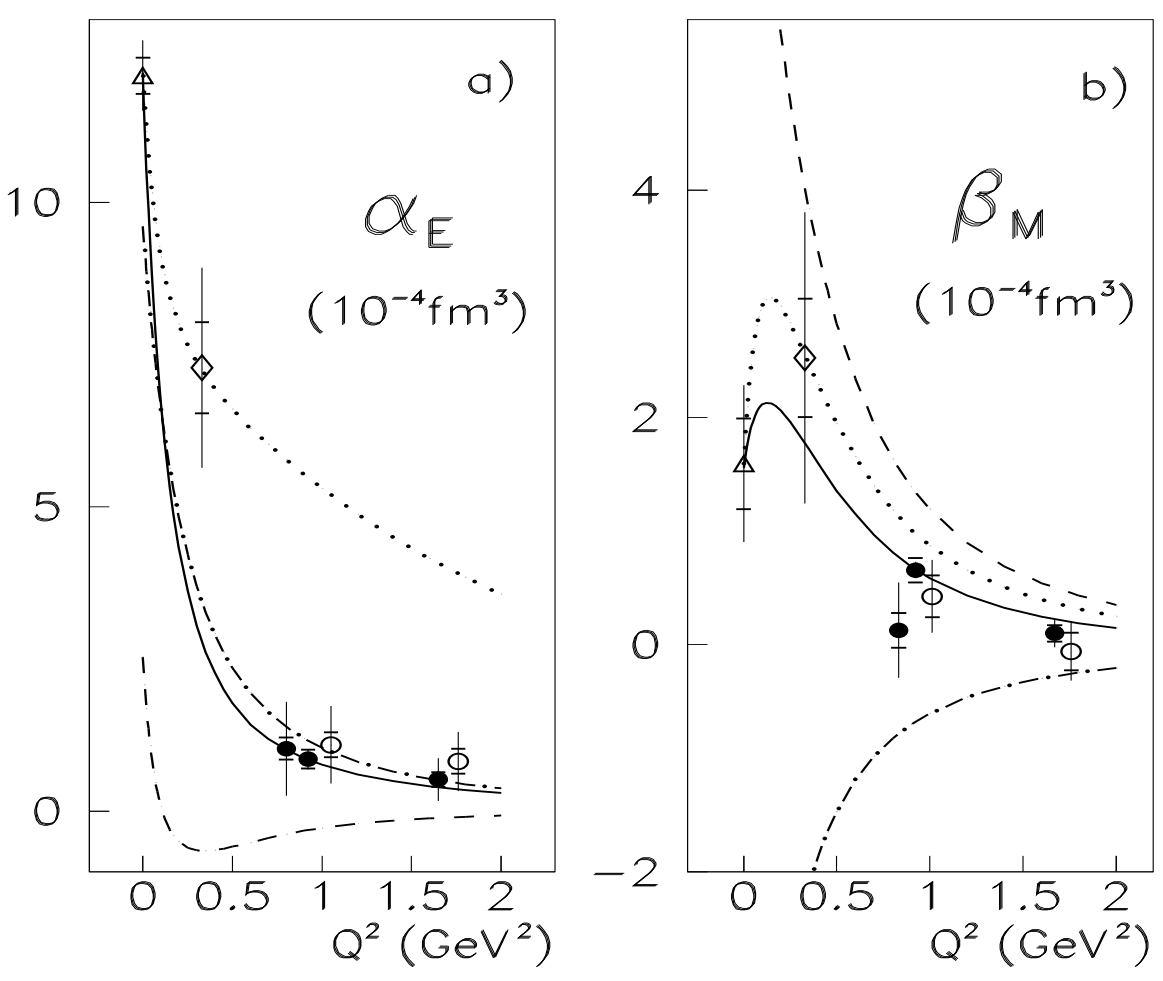

Figure 7: Compilation of the data on electric (a) and magnetic (b) GPs of the proton. Data points at $Q^{2}=0$ are from Ref. [16] $(\triangle)$. The other points are the LEX analysis of MAMI [17] $(\diamond)$ and the LEX $(\circ)$ and DR $(\bullet)$ analyses of JLab [18. Some JLab points are shifted in abscissa for better visibility. The inner error bar is statistical; the outer one is the total error. The curves show calculations in the DR model, as explained in the text.

The curves on figure 7 are calculated using the DR model. The solid curve is the DR calculation for $\Lambda_{\alpha}=0.70 \mathrm{GeV}$ and $\Lambda_{\beta}=0.63 \mathrm{GeV}$, as fitted on one of the JLab data sets. The dotted curve is the DR calculation for $\Lambda_{\alpha}=1.79 \mathrm{GeV}$ and $\Lambda_{\beta}=0.51 \mathrm{GeV}$, which reproduces the MAMI LEX point. By definition (cf. eq 5) all DR curves are constrained to go through the experimental RCS point at $Q^{2}=0$.

The fact that there is no unique DR curve going through all the data points, especially for the electric polarizability, does not invalidate the model. It simply means that the dipole parametrization of eq. 5 does not hold over the entire range of $Q^{2}$. Another fact to be aware of is the model-dependency introduced in this figure by transforming the LEX result (structure functions) into GPs. For example, the structure function $P_{T T}$ involved in this operation behaves quite differently according to different theoretical predictions [10, 6] and has convergence problems in ChPT [6, 7]. Its separate measurement would be of great interest.

In fig. 7 the DR calculation corresponding to the solid curve has been split into its $\pi N$ part (dashed) and asymptotic part (dot-dashed). While the electric polarizability is dominated by the asymptotic part, for the magnetic polarizability both contributions are important. The $\pi N$ contribution to $\beta_{M}$ is strongly paramagnetic, predominantly arising from the $\Delta(1232)$ resonance. The asymptotic part of $\beta_{M}$ is strongly diamagnetic and associated to $\sigma$-meson $t$-channel exchange. The interplay results in a 
turn-over of $\beta_{M}$ at small $Q^{2}$, as already mentioned in section 1.1] The Bates experiment 20] performed at $Q^{2}=0.05 \mathrm{GeV}^{2}$ (analysis in progress) will give new insight on this specific behavior of $\beta_{M}$.

\subsection{VCS in the resonance region}

In the JLab experiment E93-050, photon electroproduction in the resonance region was investigated for the first time, and cleanly separated from the $\left(e p \rightarrow e p \pi^{0}\right)$ channel. Cross sections have been measured up to $\mathrm{W}=1.9 \mathrm{GeV}$, at $Q^{2}=1 \mathrm{GeV}^{2}$ and backward angle $\theta_{C M}$ [19]. In figure 8 resonant structures are clearly visible up to $\mathrm{W}=1.7 \mathrm{GeV}$. At higher $\mathrm{W}$, this data together with existing large-angle RCS data show a transition to a $Q^{2}$-independent regime, suggesting that the VCS process starts coupling to elementary quarks.

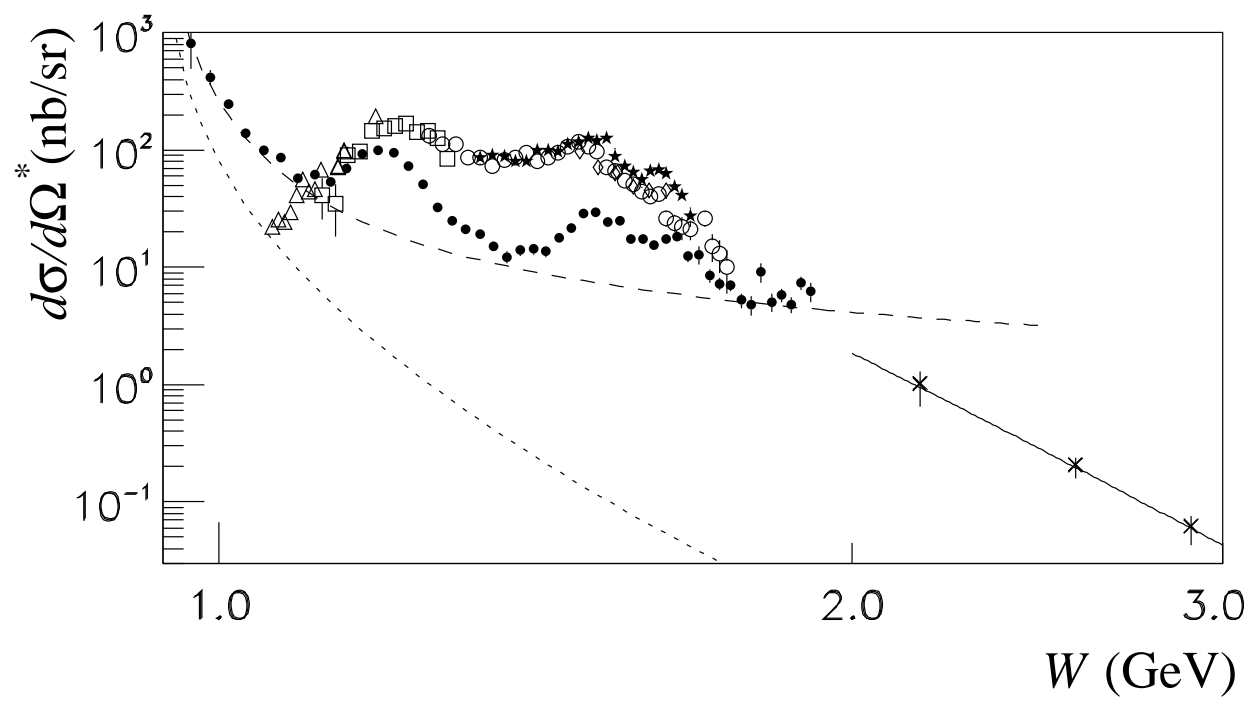

Figure 8: VCS and RCS in the resonance region. Photon electroproduction cross section data of JLab experiment E93-050 divided by the virtual photon flux factor (black dots), compared to various large angle RCS data: $(\star)$ [42, ( $\diamond)$ [4], $(\triangle)$ [4], (०) 45], (box) 46], (×) [47]). The solid curve is an $s^{-6}$ power law normalized to the $W=2.55 \mathrm{GeV}$ RCS Cornell point. The dashed curve is the $\mathrm{BH}+\mathrm{Born}+\pi^{0}$-exchange cross section and the dotted curve the $\mathrm{BH}$ alone.

\section{Present and Perspectives}

The unpolarized VCS program can be pursued along several directions:

- a more detailed mapping of the structure functions $\left(P_{L L}-P_{T T} / \epsilon\right)$ and $P_{L T}$ versus $Q^{2}$

- a Rosenbluth-type separation of the structure functions $P_{L L}$ and $P_{T T}$ by cross section measurements at different $\epsilon$

- investigate in more detail photon electroproduction in the resonance region up to $\mathrm{W}=2 \mathrm{GeV}$, in a larger phase space and with better statistics (there has been only one exploratory experiment)

- in the region of the $\Delta(1232)$ resonance, use the DR formalism to measure the electric and magnetic GPs at high $Q^{2}$ (up to $4 \mathrm{GeV}^{2}$ in the proposed experiment of ref. [26]).

Quite obviously, polarization degrees of freedom open up new possibilities of investigations in VCS. At present time there are two well-identified cases: 
Single polarization: $\quad \overrightarrow{e p} \rightarrow e p \gamma \quad$ with longitudinally polarized incoming electrons

Double polarization $\overrightarrow{e p} \rightarrow e \vec{p} \gamma \quad$ by adding the recoil proton polarization measurement.

Such a polarized VCS program is under way at Mainz and is summarized below.

\subsection{VCS with single polarization}

With a longitudinally polarized electron beam one can study the beam spin asymmetry or electron single spin asymmetry (SSA) in the $\overrightarrow{e p} \rightarrow e p \gamma$ process:

$$
S S A=\frac{d^{5} \sigma(+)-d^{5} \sigma(-)}{d^{5} \sigma(+)+d^{5} \sigma(-)}=\frac{\Delta \sigma}{2 \cdot d^{5} \sigma_{\text {unpol. }}}
$$

where $d^{5} \sigma(+)$ (resp. $d^{5} \sigma(-)$ ) is the cross section for incoming electrons of helicity $+\frac{1}{2}$ (resp. $-\frac{1}{2}$ ). Rewriting eq. 1 1 as: $T_{e p \rightarrow e p \gamma}=T_{B H}+T_{V C S}$, with the Born part now in $T_{V C S}$, the $(e p \rightarrow e p \gamma)$ cross section can be expressed as the sum of three contributions:

$$
d^{5} \sigma=\left|T_{B H}\right|^{2}+\left|T_{V C S}\right|^{2}+2 \operatorname{Re}\left(T_{B H} \cdot T_{V C S}\right)=d^{5} \sigma_{B H}+d^{5} \sigma_{V C S}+d^{5} \sigma_{\text {Interf.BH-VCS }}
$$

In the singly polarized case one can write the numerator of the SSA as:

$$
\Delta \sigma=\Delta \sigma_{B H}+\Delta \sigma_{V C S}+\Delta \sigma_{\text {Interf.BH-VCS }}
$$

The first term $\Delta \sigma_{B H}$ is zero. For the second term $\Delta \sigma_{V C S}$ we are in a case analogous to the usual decomposition of the hadron electroproduction cross section, including the fifth response function, i.e.:

$$
d^{5} \sigma_{V C S}=\Gamma_{v} \cdot\left[d \sigma_{T}+\epsilon d \sigma_{L}+\sqrt{2 \epsilon(1+\epsilon)} d \sigma_{L T} \cos \varphi+\epsilon d \sigma_{T T} \cos (2 \varphi)+h \sqrt{2 \epsilon(1-\epsilon)} d \sigma_{L T}^{\prime} \sin \varphi\right]
$$

where $\Gamma_{v}$ is the virtual photon flux and $h$ the electron beam polarization. As shown in ref. [27], the $\sigma_{L T}$ term measures the real part of the longitudinal-transverse interference, whereas the $\sigma_{L T}^{\prime}$ term measures its imaginary part, i.e. the relative phase between the longitudinal and transverse VCS helicity amplitudes. By flipping the helicity of the incoming electron, one isolates in $\Delta \sigma_{V C S}$ the fifth response function $\sigma_{L T}^{\prime}$. On the other hand, the third term of eq. 8 is usually the dominant one, in other words the SSA is mainly due to the interference of the real BH+Born amplitude with the imaginary part of the VCS amplitude [10]. One should note that this term does not necessarily behave like $\sin \varphi, \operatorname{contrary}$ to the second term $\Delta \sigma_{V C S}$. The single spin asymmetry is one case where the interference of VCS with Bethe-Heitler is not undesirable. Indeed it serves as an amplifier of the measured asymmetry; the SSA would be much smaller in the case of VCS alone.

The SSA is non-zero only for kinematics above pion threshold (because one needs an $\operatorname{Im}\left(T_{V C S}\right)$ ) and for out-of-plane values of $\varphi$. By measuring this observable, one will test the models specifically in their prediction of $\operatorname{Im}\left(T_{V C S}\right)$. Namely, in the DR model this tests the contribution of the $\pi N$ intermediate states (calculated using MAID pion photoproduction multipoles).

The MAMI single polarization VCS experiment [28]: data was taken between 2002 and 2004, at $Q^{2}=0.33 \mathrm{GeV}^{2}, \mathrm{~W}=1.2 \mathrm{GeV}, \epsilon=0.48$ and forward $\theta_{C M}$, with the proton spectrometer tilted out-ofplane. In these kinematics, the DR model predicts an SSA which is almost a pure $\sin \varphi$, with a sizeable amplitude of about $10 \%$ [11. The measurement of this amplitude will be a very specific cross-check of the dispersion formalism for VCS. The analysis of the experiment is in progress.

The experiment will also measure the SSA for the $e p \rightarrow e p \pi^{0}$ channel in the same kinematics, allowing further tests of the theory related to the fifth response function of pion electroproduction. 
The SSA in the $(e p \rightarrow e p \gamma)$ channel, as predicted by the DR model is little sensitive to the GPs for the Mainz kinematics [11. However one can analyze the experiment by doing a helicity sum and performing an unpolarized analysis of the DR type, as explained in section 1.5, to extract the electric and magnetic GPs.

Link with VCS at higher energy: The SSA in exclusive photon electroproduction at higher energy has been studied in ref. [27]. The SSA in the Deep VCS regime (DVCS) has been studied in numerous papers 29]. It is worth recalling that in DVCS this single spin asymmetry or its numerator $\Delta \sigma$ is one of the main tools to investigate the Generalized Parton Distributions (GPDs). ${ }^{5}$

\subsection{VCS with double polarization}

This is an ideal case, since the process $\overrightarrow{e p} \rightarrow e \vec{p} \gamma$ allows in principle to disentangle the six lowest order GPs. The full formalism of double polarization observables below pion threshold has been worked out in ref. [30]. One uses a longitudinally polarized electron beam and measures the polarization components $P_{x}, P_{y}, P_{z}$ of the recoil proton in the $\gamma p$ center-of-mass. The double spin asymmetry along axis $i(i=x, y, z)$ is given by:

$$
D S A_{(i)}=\frac{\left[d^{5} \sigma_{h=+1 / 2, s_{i}^{\prime} \uparrow}-d^{5} \sigma_{h=+1 / 2, s_{i}^{\prime} \downarrow}\right]-\left[d^{5} \sigma_{h=-1 / 2, s_{i}^{\prime} \uparrow}-d^{5} \sigma_{h=-1 / 2, s_{i}^{\prime} \downarrow}\right]}{\left[d^{5} \sigma_{h=+1 / 2, s_{i}^{\prime} \uparrow}+d^{5} \sigma_{h=+1 / 2, s_{i}^{\prime} \downarrow}\right]+\left[d^{5} \sigma_{h=-1 / 2, s_{i}^{\prime} \uparrow}+d^{5} \sigma_{h=-1 / 2, s_{i}^{\prime} \downarrow}\right]}
$$

where $h$ is the incoming electron helicity and $s_{i}^{\prime}$ the projection of the recoil proton's spin along axis $i$. This formula is equivalent to the following definition of the polarization component $P_{i}$ of the recoil proton along axis $i$, for a given helicity state $h$ of the incident electron:

$$
P_{i}=\frac{d^{5} \sigma_{h, s_{i}^{\prime} \uparrow}-d^{5} \sigma_{h, s_{i}^{\prime} \downarrow}}{2 d^{5} \sigma_{\text {unpol. }}}=\frac{\Delta \sigma_{h, i}}{2 d^{5} \sigma_{\text {unpol. }}}
$$

The polarization components $P_{i}$ receive contributions from $(\mathrm{BH}+\mathrm{VCS}$ Born) and (VCS NonBorn), and similarly to the unpolarized case a Low Energy Theorem can be established. This yields the following equations:

$$
\Delta \sigma_{h, i}=\Delta \sigma_{h, i}^{B H+B o r n}+\phi \cdot q_{c m}^{\prime} \cdot \Delta \Psi_{0(h, i)}+\mathcal{O}\left(q_{c m}^{\prime 2}\right)
$$

where the first term is entirely calculable, and the second term $\Delta \Psi_{0(h, i)}$ is a linear combination of the following structure functions [3] :

$$
\begin{aligned}
& \Delta \Psi_{0(h, z)}=4 h \times\left[c_{1}^{z} \cdot P_{T T}+c_{2}^{z} \cdot P_{L T}^{z}+c_{3}^{z} \cdot P_{L T}^{\prime z}\right] \\
& \Delta \Psi_{0(h, x)}=4 h \times\left[c_{1}^{x} \cdot P_{L T}^{\perp}+c_{2}^{x} \cdot P_{T T}^{\perp}+c_{3}^{x} \cdot P_{T T}^{\prime \perp}+c_{4}^{x} \cdot P_{L T}^{\prime \perp}\right] \\
& \Delta \Psi_{0(h, y)}=4 h \times\left[c_{1}^{y} \cdot P_{L T}^{\perp}+c_{2}^{y} \cdot P_{T T}^{\perp}+c_{3}^{y} \cdot P_{T T}^{\prime \perp}+c_{4}^{y} \cdot P_{L T}^{\prime \perp}\right]
\end{aligned}
$$

The $c_{n}^{i}$ are kinematic coefficients. The structure functions of eq. 13 are themselves linear combinations of the six lowest order GPs. Apart from the formulas already given in eq. 4, the new expressions for the doubly polarized case are [3] :

\footnotetext{
${ }^{5}$ in DVCS experiments, $\Delta \sigma$ is fitted to the form: $A \sin \varphi+B \sin 2 \varphi$ with $A \gg B$. The full theoretical expression of $\Delta \sigma$ contains a third term $(C \sin 3 \varphi)[48$.
} 


$$
\begin{aligned}
P_{L T}^{z} & =\frac{3 \tilde{Q} q_{c m}}{2 \tilde{q}_{0}} G_{M} P^{(01,01) 1}\left(q_{c m}\right)-\frac{3 M_{N} q_{c m}}{\tilde{Q}} G_{E} P^{(11,11) 1}\left(q_{c m}\right) \\
P_{L T}^{\prime z} & =-\frac{3}{2} \tilde{Q} G_{M} P^{(01,01) 1}\left(q_{c m}\right)+\frac{3 M_{N} q_{c m}^{2}}{\tilde{Q} \tilde{q}_{0}} G_{E} P^{(11,11) 1}\left(q_{c m}\right) \\
P_{L T}^{\perp} & =\frac{R}{2} \frac{G_{E}}{G_{M}} P_{T T}\left(q_{c m}\right)-\frac{1}{2 R} \frac{G_{M}}{G_{E}} P_{L L}\left(q_{c m}\right) \\
P_{T T}^{\perp} & =-\frac{q_{c m}}{2} G_{M}\left[3 P^{(11,11) 1}\left(q_{c m}\right)+\sqrt{\frac{3}{2}} P^{(11,11) 0}\left(q_{c m}\right)\right] \\
P_{T T}^{\prime \perp} & =\frac{q_{c m}}{2} G_{M}\left[3 \frac{q_{c m}}{\tilde{q}_{0}} P^{(11,11) 1}\left(q_{c m}\right)+\sqrt{\frac{3}{2}} \frac{\tilde{q}_{0}}{q_{c m}} P^{(11,11) 0}\left(q_{c m}\right)\right] \\
P_{L T}^{\prime \perp} & =\frac{3 \tilde{Q} q_{c m}}{2 \tilde{q}_{0}} G_{M}\left[P^{(01,01) 1}\left(q_{c m}\right)-\sqrt{\frac{3}{2}} \tilde{q}_{0} P^{(11,02) 1}\left(q_{c m}\right)\right]
\end{aligned}
$$

where $R=2 M_{N} / \tilde{Q}$. The GPs can be extracted from the linear systems above, provided the $P_{i}$ 's are measured over a large range in $\theta_{C M}$ (to allow enough variation of the coefficients $c_{n}^{i}$ ). The structure function $P_{L T}^{\prime \perp}$ can only be extracted by an out-of-plane measurement.

The $\mathrm{BH}+$ Born process yields large double polarization asymmetries. But the contribution of the GPs to these asymmetries is small, typically of a few percent, so it is tough to measure. A recent paper [31] reviews theoretical predictions for these double polarization asymmetries.

At MAMI the first test runs for such a second generation VCS experiment 28 took place in 2004, using the focal plane polarimeter and the longitudinally polarized beam. It's clearly a very challenging experiment, requiring high statistics and very reduced systematic errors.

\subsection{A Related Topic}

One can do Compton physics on the nucleon without doing an explicit Compton scattering experiment. For example in inclusive electron scattering $N\left(e, e^{\prime}\right) X$, one makes an extensive use of the optical theorem that relates the cross section of the process to the forward Compton scattering amplitude:

$$
\sigma\left(\gamma^{*}(\underline{q}) N \rightarrow X\right) \sim \operatorname{Im} T\left(\gamma^{*}(\underline{q}) N \rightarrow \gamma^{*}(\underline{q}) N\right)
$$

Here $\mathrm{T}$ is the forward doubly virtual Compton amplitude (VVCS), in which the initial and final virtual photons have the same four-momentum $\underline{q}$.

Most of the sum rules established in real photon processes lead to interesting results by generalization to a virtual photon. Some of them concern Generalized Polarizabilities; one example is the measurement of the Generalized Forward Spin GPs $\gamma_{0}$ and $\delta_{L T}$ of the neutron 32 . This is part of the so-called "extended GDH program" performed by scattering polarized electrons on a polarized Helium3 target: ${ }^{3} \vec{H} e\left(\vec{e}, e^{\prime}\right) X$.

The forward spin polarizability $\gamma_{0}$ is defined in real photon processes by:

$$
\gamma_{0}=\frac{1}{2 \pi^{2}} \int_{\nu_{0}}^{\infty} \sigma_{T T} \frac{d \nu}{\nu^{3}}
$$

where $\nu$ is the incident photon lab energy, $\nu_{0}$ this energy at the pion threshold, and $\sigma_{T T}=-\frac{1}{2}\left(\sigma_{3 / 2}-\sigma_{1 / 2}\right)$ the difference of photon absorption helicity cross sections. When going from a real to a virtual photon, one gets a generalized sum rule for $\gamma_{0}$, plus a sum rule defined only in the case of a virtual photon for the longitudinal-transverse spin GP $\delta_{L T}$ :

$$
\begin{aligned}
& \gamma_{0}\left(Q^{2}\right)=\frac{1}{2 \pi^{2}} \int_{\nu_{0}}^{\infty} \frac{K\left(\nu, Q^{2}\right)}{\nu} \sigma_{T T}\left(Q^{2}\right) \frac{d \nu}{\nu^{3}} \\
& \delta_{L T}\left(Q^{2}\right)=\frac{1}{2 \pi^{2}} \int_{\nu_{0}}^{\infty} \frac{K\left(\nu, Q^{2}\right)}{\nu} \sigma_{L T}\left(Q^{2}\right) \frac{1}{Q} \frac{d \nu}{\nu^{2}}
\end{aligned}
$$


$K$ is the virtual photon flux factor. These new observables have been measured up to $Q^{2}=0.9$ $\mathrm{GeV}^{2}$ [32] and compared to various ChPT predictions [33, 34] and the MAID model 35. Contrary to expectations, ChPT has difficulties reproducing the forward spin GP $\delta_{0}$ even at very low $Q^{2}(0.1$ $\mathrm{GeV}^{2}$ ). This data represent the first actual measurement of Generalized Spin Polarizabilities of the nucleon (here the neutron). Along the same lines there are results for the Generalized Baldin sum rule on $\alpha_{E}\left(Q^{2}\right)+\beta_{M}\left(Q^{2}\right)$ 36].

It must be emphasized that the Generalized Polarizabilities of this section are not the same as the ones introduced in the previous sections. In VCS we have only one virtual photon, whereas in VVCS (this section) we have two virtual photons, with identical virtuality. These two types of polarizabilities are however connected in the limit $Q^{2} \rightarrow 0$.

\section{Conclusion}

A nice overview of the history of VCS on the nucleon can be found in ref. [3]. It started as a rather unwanted contribution to radiative corrections to e- $\mathrm{N}$ scattering (proton bremsstrahlung is a VCS Born term). It regained interest in the 1990's with the hard scattering picture of QCD [37, 38. Two very innovative concepts in nucleon structure, the Generalized Polarizabilities 2 and the Generalized Parton Distributions [39 have lead to dedicated experiments in the last decade. These experiments, both at low energy (GPs) and high energy (GPDs), make full use of the performant electron accelerators and detectors, and more experimental results are expected in the near future. The theoretical front is very active too, e.g. in predicting the Generalized Polarizabilities. Recently, (double) VCS has become a rather wanted term of radiative corrections to e-N scattering, since it is proposed to explain the discrepancy between proton form factors measured by Rosenbluth and polarization transfer techniques [40, 41]. To conclude, two-photon physics seems to have a promising future in the study of nucleon structure.

\section{Acknowledgments}

I wish to thank all my VCS colleagues of the experimental and theoretical sides for their help and support, with special thanks to B. Pasquini, P. Guichon and H. Merkel for their proofreading of the manuscript. The work done within the JLab Hall A and the MAMI-A1 collaborations has provided the material of this talk.

This work was supported by DOE, NSF, by contract DE-AC05-84ER40150 under which the Southeastern Universities Research Association (SURA) operates the Thomas Jefferson National Accelerator Facility for DOE, by the French CEA and CNRS-IN2P3, the FWO-Flanders and the BOF-Gent University (Belgium) and by the European Commission ERB FMRX-CT96-0008.

\section{References}

[1] N.D'Hose, Proc. Erice School of Nuclear Physics, 21st Course, Prog. Part. Nucl. Phys. 44 (2000) 371.

[2] P.A.M. Guichon, G.Q. Liu and A.W. Thomas, Nucl. Phys. A 591 (1995) 606.

[3] P.A.M. Guichon and M. Vanderhaeghen, Prog. Part. Nucl. Phys. 41 (1998) 125.

[4] D. Drechsel, G. Knochlein, A. Metz and S. Scherer, Phys. Rev. C 55 (1997) 424.

[5] D. Drechsel et al., Phys. Rev. C 57 (1998) 941. 
[6] T. Hemmert, B. Holstein, G. Knochlein and D. Drechsel, Phys. Rev. D 62 (2000) 014013.

[7] C.W. Kao and M. Vanderhaeghen, Phys. Rev. Lett. 89 (2002) 272002.

[8] A. Metz and D. Drechsel, Z. Phys. A 356 (1996) 351; Z. Phys. A 359 (1997) 165.

[9] M. Vanderhaeghen, Phys. Lett. B 368 (1996) 13.

[10] B. Pasquini et al., Eur. Phys. J. A 11 (2001) 185.

[11] D. Drechsel, B. Pasquini and M. Vanderhaeghen, Phys. Rept 378 (2003) 99.

[12] G.Q. Liu, A.W. Thomas and P.A.M. Guichon, Austral. J. Phys. 49 (1996) 905.

[13] B. Pasquini, S. Scherer and D. Drechsel, Phys. Rev. C 63 (2001) 025205.

[14] F.E. Low, Phys. Rev. 110 (1958) 974.

[15] D. Drechsel, O. Hanstein, S. Kamalov and L. Tiator, Nucl. Phys. A 645 (1999) 145.

[16] V. Olmos de León et al., Eur. Phys. J. A 10 (2001) 207.

[17] J. Roche et al., Phys. Rev. Lett. 85 (2000) 708.

[18] G. Laveissiere et al., Phys. Rev. Lett. 93 (2004) 122001.

[19] G. Laveissiere et al., hep-ex/0406062, submitted to PRL.

[20] J. Shaw, R. Miskimen et al., MIT-Bates Proposal E97-03 (1997).

[21] L. Van Hoorebeke et al. (to be published in NIM).

[22] M. Vanderhaeghen et al., Phys. Rev. C 62 (2000) 025501.

[23] T. Hemmert, B. Holstein, G. Knochlein and S. Scherer, Phys. Rev. D 55 (1997) 2630.

[24] T. Hemmert, B. Holstein, G. Knochlein and S. Scherer, Phys. Rev. Lett. 79 (1997) 22.

[25] E.J. Brash, A. Kozlov, S. Li and G. Huber, Phys. Rev. C 65 (2002) 051001.

[26] JLab Hall A proposal PR-03-010, http://www.jlab.org/exp_prog/proposals/03/PR03-010.pdf

[27] P. Kroll, M. Schurmann and P. Guichon, Nucl. Phys. A 598 (1996) 435.

[28] H. Merkel and N. D'Hose, spokespersons, MAMI proposal (2000).

[29] M. Diehl, T. Gousset, B. Pire and J.P. Ralston, Phys. Lett. B 411 (1997) 193.

[30] M. Vanderhaeghen, Phys. Lett. B 402 (1997) 243.

[31] C.W. Kao, B. Pasquini and M. Vanderhaeghen, hep-ph/0408095.

[32] M. Amarian et al., Phys. Rev. Lett. 93 (2004) 152301.

[33] C.W. Kao, T. Spitzenberg and M. Vanderhaeghen, Phys. Rev. D 67 (2003) 016001.

[34] V. Bernard, T. Hemmert and U. Meissner, Phys. Rev. D 67 (2003) 076008. 
[35] D. Drechsel, S. Kamalov and L. Tiator, Phys. Rev. D 63 (2001) 114010.

[36] Y. Liang et al., nucl-ex/0410028, Submitted to Phys.Rev.Lett.

[37] G. Farrar and H. Zhang, Phys. Rev. D 41 (1990) 3348.

[38] The Pegasys Project, SLAC-R-377, Nov 1990.

[39] X. Ji, Phys. Rev. Lett. 78 (1997) 610.

[40] P. Guichon and M. Vanderhaeghen, Phys. Rev. Lett. 91 (2003) 142303.

[41] P. Blunden, W. Melnitchouk and J. Tjon, Phys. Rev. Lett. 91 (2003) 142304.

[42] Y. Wada et al., Nucl. Phys. B 247 (1984) 313.

[43] M. Jung et al., Zeit. Phys. C 10 (1981) 197.

[44] E.L. Hallin et al., Phys. Rev. C 48 (1993) 1497.

[45] T. Ishii et al., Nucl. Phys. B 254 (1985) 458.

[46] F. Wissmann et al., Nucl. Phys. A 660 (1999) 232.

[47] M.A. Shupe et al., Phys. Rev. D 19 (1979) 1921.

[48] M. Diehl, DESY-Thesis-2003-018, hep-ph/0307382. 